\title{
Folate status and colorectal cancer risk: a 2016 update
}

Joel B. Mason, M.D. (corresponding author)

Professor of Medicine and Nutrition, Tufts University; U.S.D.A. Human Nutrition Research Center at Tufts University

711 Washington St

Boston, MA 02111

617-556-3194/joel.mason@tufts.edu

San Yuan Tang, M.D.

Chief physician, Internal Medicine-Oncology, The First Affiliated Hospital, University of South China, Henyang, People's Republic of China; and Visiting Scientist, U.S.D.A. Human Nutrition Research Center at Tufts University

Abbreviations. CRC: colorectal cancer; DFE: dietary folate equivalent; MTHFR: methylenetetrahydrofolate reductase; NTD: neural tube defect 


\section{Abstract}

2 The consensus of epidemiologic evidence indicates that an abundant intake of

3 foodstuffs rich in folate conveys protection against the development of colorectal

4 cancer, and perhaps some other common cancers as well. Pre-clinical models

5 substantiate that the relationship is a genuinely causal one. Pre-clinical models

6 have also lent mechanistic insights into the biochemical and molecular pathways

7 by which adequate folate exposure conveys these protective effects, although

8 human studies are beginning to establish the relevance of our mechanistic

9 understanding to human cancer biology. Enhancement of genetic stability

10 appears to be a major mechanism by which folate sufficiency protects against

11 carcinogenesis. To date, the Wnt signaling cascade has been the pathway most

12 examined in this regard. The relationship between folate exposure and

13 colorectal cancer risk is a complex one, in part because a number of extrinsic

14 and intrinsic factors act as effect modifiers. This review discusses how the intake

15 of the other three B-vitamins integral to the 1-carbon pathway acts as one such

16 effect modifier. In addition, two concepts that remain matters of considerable

17 debate are whether parental intake of folate impacts on subsequent cancer risk

18 in the offspring, and whether excessive intakes of folate may have a paradoxical

19 cancer-promoting effect: observations underlying these two concepts are

20 presented as well. 


\subsection{Introduction}

26 Numerous epidemiological studies have been conducted over the past 25 years

27 that have explored the relationship between consumption of the B-vitamin, folate,

28 and the risk of developing cancer, and the overwhelming consensus of

29 observations indicates that greater dietary intake conveys protection against the

30 development of certain common cancers. The evidence has been most

31 compelling for colorectal cancer (CRC), with lesser degrees of evidence to

32 support such a connection for cancers of the breast, lung, pancreas and others

33 (reviewed in Chen et al, 2010). Pre-clinical experiments in a variety of rodent

34 models of intestinal cancer have substantiated the conclusions of these clinical

35 studies and proven, at least within the context of animal models, a true causative

36 role for inadequate folate intake as an independent risk factor for CRC (reviewed

37 in Kim Yl, 2003).

38 The weight of evidence is quite strong in this regard and therefore the

39 reigning opinion among investigators in this field is that habitually inadequate

40 folate consumption is a genuine independent risk factor for CRC. This

41 conclusion was reiterated by the largest prospective cohort study to examine this

42 issue to date, in which $\sim 525,000$ adults were followed for over eight years, and

43 individuals consuming the greatest quantity of total folate versus those

44 consuming the lowest were found to enjoy a $30 \%$ smaller risk of developing CRC

45 (hazard ratio=0.70; 95\% Cl: 0.58-0.84) (Gibson et al, 2011). 
However, not every clinical study on this topic has concluded that higher

47 folate status conveys protection: a few notable studies have reported either a null

48 effect of folate status on CRC risk (Eussen et al, 2010) or, curiously, a protective

49 effect of low folate status (Lee et al, 2012; Gylling et al, 2014). The absence of a

50 protective effect of higher folate status in these studies has yet to be explained,

51 although it is probably not a coincidence that each study that has failed to

52 demonstrate a protective effect of higher folate status has utilized blood levels as

53 the metric of folate exposure rather than oral consumption of the vitamin. One

54 potential explanation for these discordant results is that the studies that have

55 used blood concentrations to assess folate exposure tend to encompass much

56 smaller cohorts than those that have used dietary intake, and therefore may lack

57 sufficient power to detect a beneficial effect of folate. However, even in the

58 context of a single study where both measures of folate exposure were examined

59 there was a null association between serum folate and CRC risk but a quite

60 robust protective effect with increasing levels of dietary intake (Glynn et al, 1996).

61 This raises the question whether established modifiers of serum folate

62 concentrations such as smoking (Pfeiffer et al, 2015) and obesity (Bird et al,

63 2015) might complicate and obscure its relationship to CRC. Further, the serum

64 concentration of folate reflects the consumption of both natural sources of folate

65 as well as the pharmaceutical form of the vitamin, folic acid. This may be of

66 importance since at least one meta-analysis of prospective cohort studies

67 concluded that only natural sources of folate, and not folic acid, convey a

68 protective effect against CRC (Sanjoaquin et al, 2005). 
Several other interesting facets of the relationship between folate

70 exposure and the risk of $\mathrm{CRC}$ have emerged, and three are discussed in this

71 review: 1) the modifying effects of the other 1 -carbon vitamins, i.e.: B2, B6, and

$72 \mathrm{~B} 12 ; 2)$ the possibility of a 'transgenerational effect', in which maternal intake of

73 folate and other B vitamins during the periconceptional period, pregnancy and

74 lactation might impact on the offspring's likelihood of developing cancer later in

75 life. Indeed, there is even speculation that preconceptional paternal intake of

76 folate might impact on spermatogenesis in a manner that modulates cancer risk

77 in offspring, and 3) the concept of the so-called 'dual effect of folate' on cancer

78 risk, whereby both inadequate as well as excessive intake of folate increase

79 cancer risk when compared to adequate intake. The modulation of CRC risk

80 associated with various polymorphisms in genes that encode enzymes that play

81 roles in 1-carbon metabolism, especially the C677T single nucleotide

82 polymorphism (SNP) in methylenetetrahydrofolate reductase (MTHFR), has

83 recently been reviewed (Figueiredo et al, 2013) and will therefore not be

84 discussed in depth in this review.

85

\subsection{Scientific Background}

87 As mentioned above, the consensus of over 30 case-control and prospective

88 cohort studies indicate that individuals who habitually consume the greatest

89 quantities of dietary folate have a $20-60 \%$ reduction in the risk of developing

90 colorectal cancer as well as a similar reduction in the risk of having the precursor

91 lesion, the adenomatous polyp (Chen et al, 2010). Moreover, multiple studies 
92 indicate that the protective effect is more pronounced among those who are

93 moderate-to-heavy consumers of alcohol (Giovannucci, 2004; Gibson et al,

94 2011), a known inhibitor of folate metabolism (Mason and Choi, 2005). Not

95 surprisingly, a meta-analysis of the prospective cohort studies has confirmed the

96 protective effect of higher folate intake against the development of colorectal

97 cancer (Sanjoaquin et al, 2005). Although one must draw conclusions regarding

98 dosage cautiously from epidemiologic studies, the largest study to date would

99 suggest that $\sim 500$ micrograms of total folate per day conveys the maximal

100 protection (Gibson et al, 2011). The latter study did not report folate

101 consumption in terms of dietary folate equivalents (DFEs), which corrects for the

102 differential in bioavailability between folic acid and folate derived from food, so for

103 now the apparent optimal amount is necessarily described in terms of total

104 micrograms of the various forms of folate.

105 Several lines of evidence support a true mechanistic role for folate in the

106 prevention of cancer. First, controlled studies in a number of animal models

107 have demonstrated a dose-dependent protective effect of folate (reviewed in Kim

$108 \mathrm{YI}, 2004)$. Second, the fact that homozygous carriers of the common C677T

109 polymorphism of methylenetetrahydrofolate reductase have repeatedly been

110 shown to enjoy substantial protection against CRC also supports a mechanistic

111 role for folate availability in carcinogenesis (reviewed in Figueiredo et al, 2013)

112 although, interestingly, the apparent protection conveyed by this SNP largely

113 disappears among those who have low markers of folate exposure (Kennedy et

114 al, 2011). Finally, the likelihood of a true cause and effect relationship between 
115 inadequate folate intake and an increased risk of cancer is strongly supported by

116 the biological plausibility of the concept since folate is a critical co-factor in both

117 biological methylation and nucleotide synthesis (Ciappio and Mason, 2010), and

118 aberrancies of each of these two processes are thought to be among the most

119 common mechanisms leading to cancer.

120 In further support of the mechanistic role played by folate depletion in

121 colorectal carcinogenesis, studies in rodent models have identified the colonic

122 Wnt cascade as a cell signaling pathway that is upregulated by mild

123 inadequacies in folate status, especially when present in conjunction with mild

124 inadequacies of the other related 1-carbon vitamins (Liu et al, 2007; Liu et al,

125 2011). Diets mildly inadequate in folate, $B 2, B 6$, and $B 12$ have been shown to

126 produce DNA strand breaks in the $A p c$ gene, a negative regulator of Wnt

127 signaling and a four-fold increase in in vivo Wnt signaling accompanied by an

128 increase in proliferation and inhibition of apoptosis (Liu et al, 2011). Although a

129 basal level of Wnt signaling is necessary to sustain the very high rate of

130 proliferation normally present in the colonic crypt, inappropriate overactivation of

131 the pathway due to genetic or epigenetic aberrations is found in over $80 \%$ of

132 human colorectal cancers and is considered one of the earliest 'gatekeeper

133 events' that enables normal colonocytes to follow a pathway towards neoplastic

134 transformation. Lesser evidence has also implicated aberrations in the p53

135 pathway as possibly playing a role as well (Liu et al, 2008).

136 It is rather tempting to also invoke anomalies in the methylation of DNA,

137 and other methylated biomolecules as a mechanism by which folate inadequacy 
138 promotes carcinogenesis but accruing evidence suggests this not to be true.

139 Even rather severe degrees of folate depletion do not predictably induce aberrant

140 methylation in the exons or promoter regions of critical tumor suppressor genes

141 (Sohn et al, 2003). Also, biochemical 'rescue' of the methylation cycle within

142 folate metabolism has been shown to not concur with reductions in tumorigenesis

143 (Knock et al, 2008), whereas enriching the activity of cytosolic serine

144 hydroxymethyltransferase, which operates on the nucleotide synthetic side of the

145 folate metabolism cycle, markedly reduces tumorigenesis (McFarlane et al,

146 2014). The possible exception is the effect of maternal folate intake on cancer

147 risk in offspring, where evidence is emerging that aberrations in DNA methylation

148 might convey the transgenerational effect on cancer risk (see below).

149 Five prospective, randomized intervention trials have been conducted

150 among individuals with previously resected colonic adenomas (Cole et al, 2007;

151 Paspatis and Karamanolis, 1994; Jaszewski et al, 2008; Logan et al, 2008; Wu et

$152 \mathrm{al}$, 2009) in order to determine whether daily administration of folic acid

153 supplements, at doses varying from $0.5-5 \mathrm{mgs} /$ day, prevents the appearance of

154 subsequent adenomas (adenomas, especially of the 'high-risk' variety are

155 considered valid intermediary biomarkers of CRC risk (Schatzkin, 2000). The two

156 trials considered to be the weakest (due to either their small size [Paspatis and

157 Karamanolis, 1994] or to a less-rigorous-than-desirable study design [Jaszewski

158 et al, 2008]) each reported a significant protection against adenoma development

159 with folic acid supplementation. However, the meta-analysis of the three trials

160 whose designs are considered to be the most scientifically robust is probably 
161 more informative and accurate ( $n=2632$ subjects in total): this analysis found that

162 folic acid supplementation did not convey protection against adenoma

163 occurrence in the subject population as a whole, although among those whose

164 underlying plasma folate was in the lowest quartile at the onset of intervention a

165 marginally significant $20 \%$ decline in the relative risk of adenoma occurrence was

166 enjoyed among those receiving folic acid (Figueiredo et al, 2011). This is

167 consistent with our present understanding, which is that adequate amounts of

168 folate intake are protective compared to inadequate intake, but supplementation

169 of those who are already folate-replete conveys no additional protection against

170 CRC.

171 The evidence implicating a protective role for adequate folate status in

172 other cancers is not as compelling, in part because the issue has not yet been as

173 extensively examined, and because there is not a consistency of results among

174 the studies. Nevertheless, there are observations implicating a protective role for

175 adequate folate intake in human cancers of the oropharynx, esophagus,

176 stomach, pancreas, lungs, cervix, ovary, breast, neuroblastoma and leukemia

177 (Larsson, et al, 2006; Lewis et al, 2006; Larsson et al, 2007).

178

179 3.0 One-carbon vitamins other than folate as determinants of cancer risk

180 Folate is not the sole dietary component that operates as a critical co-factor in

181 the network of biochemical reactions that is typically referred to as "1-carbon

182 metabolism'. Consequently, assessing the impact of diet on the cellular integrity

183 of 1-carbon metabolism by measuring folate status alone is often misleading 
184 because vitamins $\mathrm{B} 2, \mathrm{~B} 6$, and $\mathrm{B} 12$ serve as critical co-factors for several pivotal

185 enzymes as well. Further, other dietary components such as methionine and

186 choline are important substrates in 1-carbon metabolism as well. Consequently,

187 biochemical functions performed by 1 -carbon metabolism are often modified by

188 the availability by several of these dietary factors. For instance, the disposal of

189 homocysteine (which ultimately determines plasma homocysteine

190 concentrations) reflects a complex 3-way interaction between folate, B12, and B6

191 status (Selhub et al, 1996). Moreover, since riboflavin is a co-factor for MTHFR,

192 plasma riboflavin is also a determinant of plasma homocysteine among those

193 who carry the C677T MTHFR polymorphism (Hustad, et al, 2000). Mathematical

194 modeling of the known enzyme kinetics of 1-carbon metabolism reveals that

195 critical functions of the pathway, such as nucleotide synthesis, are a complex

196 function of the availability of all four of these vitamins (Reed et al, 2006). The

197 inter-connectedness of these four vitamins is similarly important to those

198 metabolic outcomes that are relevant to carcinogenesis: for example, limiting B12

199 availability alone in a rodent model alters uracil incorporation in colonic DNA, a

200 function of 1-carbon metabolism that is 'distant' from the methionine synthase

201 reaction that B12 participates in (Choi et al, 2004). In addition, rodent studies

202 demonstrate that many of the pro-transformational molecular events that occur

203 as a result of altered 1-carbon metabolism, such as upregulation of Wnt

204 signaling, are greatly magnified when inadequacies of multiple 1-carbon

205 micronutrients are present compared to folate depletion alone (Liu et al, 2007;

206 Liu et al, 2011). A thorough mechanistic understanding of how folate modulates 
207 carcinogenesis should therefore include an examination all four 1-carbon 208 vitamins.

209 Clinical observations also provide a compelling argument for why all of the

210 1-carbon micronutrients should be examined in combination in regard to their

211 impact on cancer risk. First, an increasing number of epidemiological studies

212 have observed an association between low vitamin B6 and B12 status with an

213 increased risk of common neoplasms (Lajous, et al, 2006; Zhang et al, 2003; Wu

214 et al, 1999, Johansson et al, 2010), and these relationships persist even when

215 the effects of folate blood levels or intake are regressed out of the relationship.

216 Second, the study of these inadequacies is relevant to adult populations in the

217 developed world: although flagrant deficiencies of these vitamins are uncommon

218 in industrialized societies, marginal status, which is to say biochemical measures

219 of vitamin status below the accepted normative range but in the absence of signs

220 or symptoms of the classical deficiency syndromes, are rather common.

221 Population-based studies have reported that $18-25 \%$ of adults have low B6

222 status and $10-20 \%$ of healthy elders are thought to have 'subclinical' B12

223 deficiency (Planells, et al, 2003; Lindenbaum, et al, 1994). Further, a recent

224 nutrition survey of the United Kingdom reveals that $>50 \%$ of adults have

225 subnormal levels of the most accurate blood marker of riboflavin status (National

226 Diet and Nutrition Survey, 2004).

227

228 4.0 Might maternal folate consumption impact on cancer risk in offspring? 
229 Maternal nutrition is becoming increasingly recognized as a determinant of

230 chronic disease in offspring (Barker, 2004), and this principle may apply to both

231 macronutrients as well as select micronutrients. Although it remains speculative

232 as to whether a genuine effect exists in the human population, there are a

233 number of observations suggesting that a mother's habitual intake of folate pre-

234 conceptionally, during pregnancy, and perinatally might impact on the risk of

235 cancer in offspring. Epidemiological evidence does exist to support a protective

236 role for high maternal folate intake against certain pediatric cancers in offspring,

237 including retinoblastoma (French et al, 2003; Goh et al, 2007), non-Hodgkin's

238 lymphoma (Schuz et al, 2007), acute lymphoblastic leukemia (Thompson et al,

239 2001) and childhood brain tumours (Goh et al, 2007), although, in balance, there

240 is some inconsistency since some epidemiologic studies have not been able to

241 observe such protection (Milne et al, 2010; Ajrouche et al, 2014; Chokkalingam

242 et al, 2013; Orjuela et al, 2012). It is likely that the cellular pathways by which

243 folate is thought to exert its impact on cancer risk in adults, as was described

244 above, are also operable in the embryo, fetus and newborn. Moreover, although

245 anomalies in DNA methylation due to folate inadequacy have not been

246 convincingly shown to be instrumental in modulating cancer risk in adults (e.g.

247 Sohn et al, 2003; Knock et al, 2008; McFarlane et al, 2014), the evolving fetus

248 may be particularly susceptible to the establishment of aberrant patterns of

249 methylation since marked swings in DNA methylation occur during

250 embryogenesis ( $\mathrm{Li}, 2002)$ and cells are dividing rapidly. Evidence that maternal

251 folate intake can impact on the offspring's epigenome comes from studies with 
252 'Agouti' (Avy/a) mice in which maternal supplementation with folate and related

253 dietary methyl donors resulted in a shift in the offspring's coat color from yellow

254 to brown in conjunction with de-novo methylation of specific sequences in the

255 agouti gene (Cooney et al, 2002; Waterland and Jirtle, 2003) Also, maternal

256 plasma folate concentrations in humans were shown to concur with nearly 50

257 CpG sites in cord blood DNA, suggesting that a causal relationship between

258 maternal folate intake and the offspring epigenome may be relevant to human

259 biology as well (Joubert et al, 2016).

260 There are several studies in rodent models of colorectal and breast cancer

261 that support the concept that supplemental levels of folate intake in the mother

262 significantly diminishes the risk of cancer in her offspring. In a chemical

263 carcinogen model in rats, a comparison of maternal folic acid supplementation at

2642.5 times the basal requirement versus the basal requirement prior to and during

265 pregnancy, and extending into the period of lactation diminished tumorigenesis in

266 offspring by two-thirds (Sie et al, 2011). Notably, a similar level of

267 supplementation provided to the pups after weaning did not convey protection.

268 Also, the fathers received identical diets to the mothers periconceptionally, so

269 protection conveyed by paternal diet can not be ruled out. Some differences in

270 the extent of genomic DNA methylation in the colorectum of the offspring were

271 noted between the diet groups, and this was suggested as a potential

272 mechanism by which the modulation of cancer incidence was effected. Similarly,

273 in a genetically-driven model of colorectal cancer in Apc1638N mice, maternal

274 supplementation of folic acid and the other 3 related B-vitamins prior to and 
275 during pregnancy through weaning suppressed, while mild deficiencies 276 promoted, tumorigenesis in the offspring (Ciappio et al, 2011). Suppression of

277 the Wnt pathway in the intestines of the pups whose mothers received either the

278 basal or supplemental levels of B-vitamins was suggested by changes in gene

279 expression and $\beta$-catenin protein, providing a plausible mechanism by which

280 maternal B vitamin intake might be modulating tumorigenesis in the offspring.

281 There were also indications that changes in the gene expression of one of the

282 negative regulators of Wnt signaling, Sfrp1, was being altered by changes in

283 promoter methylation of that gene, providing further support to the theory that

284 changes in DNA methylation might be a mechanism by which transgenerational

285 transmission of risk is conveyed.

286 Considerably more speculative is the idea that paternal folate intake prior 287 to conception might, to some extent, determine cancer risk in the next 288 generation. In support of this idea are observations in mice that paternal folate 289 depletion can lead to changes in the epigenome of sperm, and increased birth 290 defects in the offspring (Lambrot et al, 2013). A single study comparing paternal

291 B vitamin supplementation, repletion and depletion has been conducted in the 292 Apc1638N model in which the endpoint of offspring intestinal tumorigenesis was

293 examined. No overt changes in tumor incidence or multiplicity were observed 294 although mean tumor volume increased in the female offspring in a stepwise 295 fashion with increasing paternal B vitamin intake (Sabet et al, 2016).

296 Three rodent studies have examined whether maternal folate intake 297 impacts on the risk of mammary cancer and the results are discordant. In one 
298 study maternal folic acid supplementation compared to basal levels of the vitamin

299 significantly reduced an intermediary biomarker of mammary cancer risk in

300 female offspring, terminal end buds (Sie et al, 2009). Similarly, maternal

301 supplementation of folate, B12, choline and methionine at five times that present

302 in the basal diet reduced carcinogen-induced mammary carcinogenesis in female

303 offspring (Cho et al, 2012). In contrast, maternal folic acid supplementation

304 increased the risk of carcinogen-induced mammary tumors in the female

305 offspring of rat dams (Ly et al, 2011). The inconsistency of results between

306 these three studies makes it difficult to draw conclusions: perhaps the most

307 important lesson to be learned is that different experimental protocols asking a

308 similar research question can produce very different results, underscoring the

309 need to determine which animal models are most relevant to human cancer

310 biology and what details of a study protocol are the critical determinants of the

311 outcomes.

313 5.0 The potential for cancer promotion with an overly-abundant intake of 314 folate

315 Folate's central role as a co-factor in nucleotide synthesis means that abundant 316 availability of the vitamin can facilitate the proliferation of rapidly dividing cells,

317 and hyperproliferation is a feature of most dysplastic ('pre-cancerous') and 318 malignant ('cancerous') neoplasms. The earliest evidence that folate

319 consumption at levels achievable through the use of supplements might cause a 320 paradoxical acceleration of carcinogenesis dates back to the 1940s when two 
321 groups of investigators gave large doses of folic acid to patients with acute

322 leukemia and observed what Sidney Farber politely termed "the acceleration

323 phenomenon", whereby the rate of expansion of the leukemic clone increased

324 tremendously (Farber 1949; Heinle and Welch, 1948). The most intuitive

325 explanation for this phenomenon is that neoplastic cells have a high rate of

326 proliferation and therefore require large quantities of folate to maintain the

327 synthesis of thymidylate and purines at the rapid pace required to meet

328 heightened demands for DNA synthesis (Shpitz et al, 1997). However, other

329 possible mechanisms exist. For example, supplemental folic acid has been

330 reported to produce a transcriptomic environment that is pro-inflammatory in the

331 human colon (Protiva et al, 2011), and chronic low-grade inflammation is thought

332 to be a condition that pre-disposes to neoplastic transformation. Controlled

333 studies in a variety of animal models of colon cancer support the contention that

334 supplemental quantities of folic acid can promote tumorigenesis. In settings

335 where there is a particularly strong underlying predisposition to colon cancer or in

336 a setting where neoplastic tumors are already established, supplemental folic

337 acid is protective only before neoplastic foci appear in the intestine. Once such

338 foci are established, the more folic acid that is given, the faster microscopic foci

339 and macroscopic tumors arise (Song et al, 2000a; Song et al, 2000b; Kim Yl et

340 al, 1996; Leleu et al, 2000; Wargovich et al, 1996).

341 The results of a randomized trial of folic acid in the prevention of colorectal

342 adenomas among 1021 human subjects with a history of prior adenomas are

343 consistent with such an effect (Cole et al, 2007). At the time of the second 
344 follow-up, 6-8 years after initial randomization, this study observed a significant

345 2.3-fold increase in the multiplicity of recurrent adenomas among those receiving

346 folic acid supplements, as well as a marginally significant 1.7 -fold increase in so-

347 called 'high risk' adenomas. One could reasonably speculate that a sizeable

348 number of individuals with high risk neoplasms could have been enrolled in this

349 trial in spite of undergoing a baseline 'cleansing' colonoscopy since the 'miss

350 rate' for advanced adenomas at the time of colonoscopy is thought to be $\sim 10 \%$

351 (Heresbach et al, 2008), and 'flat adenomas'--which are difficult to detect by

352 conventional colonoscopy--are common and much more likely to contain foci of

353 carcinoma than polypoid adenomas (Soetikno et al, 2008). If supplemental folic

354 acid in high quantities genuinely promotes the growth of indolent neoplasms, it

355 could then have accelerated the growth of these residual adenomas.

356 To date, four other folic acid supplementation trials that have used

357 adenoma recurrence as an endpoint have been reported (Paspatis and

358 Karamanolis, 1994; Jaszewski et al, 2008; Logan et al, 2008; Wu et al, 2009) and

359 none have observed a similar increase in tumorigenesis, but importantly, none of

360 these other four have had the extended follow-up over 6-8 years that was

361 provided for in the Cole et al trial. Similarly, when Ebbing et al combined the

362 results of two randomized homocysteine-lowering trials in Norway encompassing

363 over 6800 subjects, a $21 \%$ increase in the hazard ratio of developing cancer was

364 observed among those receiving folic acid supplements (Ebbing et al, 2009). It is

365 notable, however, that stratifying the data for individual cancers identified a

366 significant increase in lung cancer rather than colorectal cancer. In an 
367 observational study, an increase of $\sim 4$ cases/100,000 individuals in the

368 nationwide rates of colorectal cancer diagnoses was reported to have

369 accompanied the first few years of mandatory folic acid fortification of cereal

370 grains in both the U.S.A. and Canada in the 1990s, and it was during the initial

371 years of these fortification programs when major millers were thought to have far

372 exceeded the mandated levels of fortification (Mason et al, 2007). Observations

373 from the abovementioned studies collectively raise a concern about the

374 possibility of a promotional effect of overly abundant intake of folate: 1) that is

375 consistent with the known biochemical functions of folate in DNA synthesis, 2)

376 that can be readily replicated in animal models of colorectal carcinogenesis, and

377 3) which is a phenomenon that has been observed to occur in both inadvertent

378 and intentional intervention trials in human subjects.

379 Moreover, organs other than the colon in which indolent foci of dysplasia

380 commonly exist in older individuals, such as the prostate, may be similarly

381 susceptible to this phenomenon. In the Cole et al trial, a secondary analysis of

382 prostate cancer incidence revealed that male subjects randomized to receive

383 folic acid supplements experienced a 2.6-fold increased incidence of clinically-

384 significant prostate cancer (Figueiredo et al, 2009). Similarly, in a meta-analysis

385 of prospective cohort studies, the odds ratio of developing prostate cancer was

$3861.18(\mathrm{p}<0.02)$ for every $10 \mathrm{nmol} / \mathrm{L}$ increase in plasma folate (Collin et al, 2010).

387 Also, in a meta-analysis of ten randomized, controlled trials in which folic acid

388 supplements were administered ( $n>38,000$ subjects) the overall incidence of

389 cancer was increased to only a marginally significant degree $(R R=1.07,95 \%$ C.I. 
390 1.00-1.14). However, when individual cancers were examined, the RR of

391 prostate cancer was $1.24(95 \%$ C.I.=1.03-1.49) (Wien et al, 2012).

392 The possibility of a paradoxical cancer-promoting effect of excessive

393 quantities of folate may be operable in human breast cancer as well: in a

394 prospective cohort study among $\sim 25,000$ postmenopausal women the risk of

395 developing breast cancer was significantly increased among those taking folic

396 acid supplements (Stolzenberg-Solomon et al, 2006); a group among whom

397 consumption was $\geq 853 \mathrm{mcg}$ of folate per day. Moreover, a promoting effect of

398 folic acid has also been observed to occur in a rodent model of mammary

399 carcinogenesis (Kotsopolous et al, 2005).

400 Nevertheless, the question as to whether excessive folate intake can 401 promote cancer in some segments of human populations continues to be a very 402 controversial topic since several null observations have been reported as well, 403 arguing against such an effect. Clarke and his colleagues (Vollset et al, 2013) 404 also performed a systematic analysis. This study included nearly 50,000 405 subjects enrolled in 13 RCTs in which the daily intervention was $\geq 500 \mathrm{mcg}$ of 406 folic acid. These investigators found a RR of cancer incidence of 1.06 (95\% C.I.

$407 \quad 0.99-1.13)$ associated with folic acid supplementation, and since the confidence 408 interval bridged 1.00 the investigators concluded no significant effect on cancer 409 incidence was likely. The data was also analyzed by the type of cancer, and 410 after correction for multiple comparisons, no individual variety of cancer 411 appeared to be increased by folic acid administration. 
412 The results of two large, prospective cohort studies have also lacked

413 evidence of a promotional effect of excessive folate intake (Stevens et al, 2011;

414 Gibson et al, 2011). These two studies each reported a significant $20-30 \%$

415 decrease in the risk of developing $\mathrm{CRC}$ among those taking the highest amounts

416 of total folate versus the lowest, with an apparent optimum for this purpose of

$417 \sim 500$ mcgs of folate from all sources per day. These two studies were large: in

418 the instance one the study population was $~ 100,000$ individuals and in the

419 second, over 500,000. Nevertheless, as previously stated (Mason, 2011),

420 neither the Gibson et al (Gibson et al, 2011) nor the Stevens et al (Stevens et al,

421 2011) studies were able to satisfactorily examine those segments of their study

422 populations that would be most susceptible to a cancer-promoting effect of high

423 folate intake, such as those ingesting $>1000$ mcgs of total folate per day or those

424 harboring indolent foci of colonic or prostate neoplasia.

425 In this era it is not at all difficult to consume $>1000$ mcgs of total folate in a

426 day. By consuming a multivitamin and a bowl of fortified ready-to-eat cereal in

427 the morning, federally mandated fortified flour in various foodstuffs during the

428 course of the day, and a B-complex tablet at night, one can easily exceed 1300

$429 \mu \mathrm{g}$ of folic acid per day (and this does not even include the $\sim 200-250$ additional

430 micrograms ingested by most adults as naturally-occuring folates).

431 Re-stated, the 'dual effect' hypothesis contends that higher intakes of

432 folate are cancer-protective in nearly all circumstances except among those

433 individuals who have existing neoplastic lesions and who are consuming

434 exceptionately large quantities of the vitamin, in which case the folate presents 
435 additional risk for tumorigenesis. Cancers that slowly evolve over years through

436 an indolent dysplastic phase, such as the colorectal adenoma or a dysplastic

437 nodule of the prostate, are exceedingly common in the general population of

438 healthy, elder adults and would be particularly prone to such an effect.

439 The fact that folic acid and not a naturally-occurring form of the vitamin is

440 used by the food and pharmaceutical industries for fortification and 441 supplementation is potentially of importance. Folic acid is converted to a natural

442 biological form of the vitamin as it passes through the intestinal wall, with

443 enzymatic reduction and methylation resulting in the circulating form of the

444 vitamin, 5-methyltetrahydrofolate. Nevertheless, oral doses of folic acid as small

445 as 200 mcgs can saturate this conversion mechanism (Sweeney et al, 2003;

446 Kelly et al, 1996; Sweeney et al, 2006; Sweeney et al, 2007), resulting in

447 detectable levels of circulating folic acid, and it is theorized that this oxidized,

448 non-substituted form of folate might feasibly be detrimental due to some

449 idiosyncratic action that does not exist for naturally-occuring forms of folate,

450 possibly because it can bypass certain regulatory nodes in the 1-carbon

451 metabolic scheme, entering as THF rather than 5-methylTHF. In the U.S., 40\% of

452 adults 60 years and older are thought to regularly consume a supplement

453 containing folic acid, most of which contain $400 \mathrm{mcg}$ per pill (Radimer et al,

454 2004), and therefore it is not surprising that a nationally representative survey

455 found that $\sim 40 \%$ of individuals over 60 had detectable levels of unmetabolized

456 folic acid in the blood (Bailey et al, 2010). Of additional concern are recent

457 animal and clinical observations indicating that high folic acid exposure might 
458 possess an idiosyncratic effect of inhibiting the cytotoxicity of circulating natural

459 killer cells (Troen et al, 2006; Sawaengsri et al, 2016). Natural killer cells are a

460 population of lymphocytes thought to play a role in the destruction of arising

461 clones of neoplastic cells. Thus, the coenzymatic form of folate used for

462 fortification is perhaps relevant in determining whether a detrimental effect is

463 produced from excessive intake, although the concern remains a theoretical one.

464 When examined in a nested case-control fashion, detectable levels of

465 unmetabolized folic acid did not predict the occurrence of colorectal cancer (Cho 466 et al, 2015).

467 The question of whether there is indeed a cancer-promoting effect of 468 excessive folate intake in the human population remains a hotly debated issue.

469 On the one hand, observations suggesting that a cancer-promoting effect has

470 been created among segments our population are rather weak, but on the other

471 hand the biological plausibility of the effect can not be denied and the large-scale

472 ramifications of such an effect, if it exists, are not ones that we can afford to 473 overlook.

\section{$475 \quad 6.0$ Conclusions}

476 In our opinion sufficient evidence has accrued to conclusively state that habitual

477 intake of adequate quantities of folate, compared to inadequate intake, sizeably

478 reduces the risk of developing colorectal cancer, and a similar effect may exist

479 for other cancers as well. There are clearly several effect modifiers such as 480 alcohol ingestion, intake of related B-vitamins, relevant genetic variants: these 
481 are factors that need to be considered when examining this topic. The question

482 of whether maternal (or even paternal) intake of folate might impact on cancer

483 risk in the subsequent generation, as well as the issue of whether folate might act

484 in a paradoxical, cancer-promoting fashion in certain segments of our society

485 remain matters of open debate. A more in-depth understanding of these

486 phenomena will be needed if we are to fully exploit these principles for the

487 purposes of cancer prevention.

\section{Funding Sources}

490 This work supported in part by the U.S. Department of Agriculture, under

491 agreements 58-1950-0-014 and 58-1950-4-003 (to JBM), and a grant from the

492 National Institute of Food and Agriculture (accession no. 1007740, to JBM). Any

493 opinions, findings, conclusions or recommendations expressed in this publication

494 are those of the author and do not necessarily reflect the views of the U.S.

495 Department of Agriculture. The authors do not have any conflicts of interest to 496 declare.

\section{References}

498 Ajrouche R, Rudant J, Orsi L, et al. (2014) Maternal reproductive history, fertility 499 treatments and folic acid supplementation in the risk of childhood acute leukemia:

500 the ESTELLE study. Cancer Causes Control 25:1283-93.

501 Bailey R, Mills J, Yetley E, et al. (2010) Unmetabolized serum folic acid and its 502 relation to folic acid intake from diet and supplements in a nationally 503 representative sample of adults aged $>$ or $=60$ y in the United States. Am. J. Clin. 504 Nutr. 92:383-9. 
505 Bird J, Ronnenberg A, Choi SW, et al. (2015) Obesity is associated with 506 increased red blood cell folate despite lower dietary intakes and serum 507 concentrations. J Nutr. 145: 79-86. doi: 10.3945.

508 Chen J, Xu X, Liu A, Ulrich C. Folate and Cancer: Epidemiological Perspective in 509 Bailey, L., Ed. (2010) Folate in Health and Disease, $2^{\text {nd }}$ edition, Taylor \& Francis 510 LLC, Boca Raton, FL, pp. 205-34.

511 Cho K, Mabasa L, Bae S, et al. (2012) Maternal high-methyl diet suppresses 512 mammary carcinogenesis in female rat offspring. Carcinogenesis 33:1106-12.

513 Cho E, Zhang X, Townsend MK, et al. (2015) Unmetabolized Folic Acid in 514 Prediagnostic Plasma and the Risk of Colorectal Cancer. J. Natl. Cancer Inst. 515 107:djv260.

516 Choi SW, Friso S, Ghandour H, et al. (2004) Vitamin B-12 deficiency induces 517 anomalies of base substitution and methylation in the DNA of rat colonic 518 epithelium. J. Nutr.134: 750-5.

519 Chokkalingam A, Chun D, Noonan E, et al. (2013) Blood levels of folate at birth 520 and risk of childhood leukemia. Cancer Epidemiol. Biomarkers Prev. 22:1088-94.

521 Ciappio E, Mason, JB, Folate and Cancer: Mechansims in Bailey, L., Ed. (2010) 522 Folate in Health and Disease, 2nd edition, Taylor \& Francis LLC, Boca Raton, 523 FL, pp. 235-62.

524 Ciappio E, Liu Z, Brooks R, et al. (2011) Maternal B vitamin supplementation 525 from preconception through weaning suppresses intestinal tumorigenesis in 526 Apc1638N mouse offspring. Gut 60:1695-702.

527 Cole B, Baron J, Sandler R, et al. Polyp Prevention Study Group. (2007) Folic 528 acid for the prevention of colorectal adenomas. JAMA 297: 2351-9.

529 Collin S, Metcalfe C, Refsum H, et al. (2010) Circulating folate, vitamin B12, 530 homocysteine, vitamin B12 transport proteins, and risk of prostate cancer: a 531 case-control study, systematic review, and meta-analysis. Cancer Epidemiol. 532 Biomarkers Prev. 19:1632-42.

533 Cooney CA, Dave AA, Wolff GL. (2002) Maternal methyl supplements in mice 534 affect epigenetic variation and DNA methylation of offspring. J. Nutr. 132(Suppl 535 8):2393S-400S.

536 Ebbing M, Bønaa KH, Nygård O, et al. (2009) Cancer incidence and mortality 537 after treatment with folic acid and vitamin B12. JAMA 302: 2119-26.

538 Eussen S, Vollset S, Igland J, et al. (2010) Plasma folate, related genetic 539 variants, and colorectal cancer risk in EPIC. Cancer Epidemiol. Biomarkers Prev. 540 2010;19: 1328-40. 
541 Farber S. (1949) Some observations on the effect of folic acid antagonists on 542 acute leukemia and other forms of incurable cancer. Blood 4: 160-7.

543 Figueiredo J, Grau M, Haile R. (2009) Folic Acid and Risk of Prostate Cancer: 544 Results From a Randomized Clinical Trial. J. Natl. Cancer Inst. 101: 432 - 435.

545 Figueiredo J, Mott L, Giovannucci E, et al. (2011) Folic acid and prevention of 546 colorectal adenomas: a combined analysis of randomized clinical trials. Int. J. 547 Cancer 129:192-203.

548 Figueiredo J, Levine A, Crott J, Baurley J, Haile R. (2013) Folate-genetics and 549 colorectal neoplasia: what we know and need to know next. Mol. Nutr. Food 550 Res. 57: 607-27.

551 French A, Grant R, Weitzman S, et al. (2003) Folic acid food fortification is 552 associated with a decline in neuroblastoma. Clin. Pharmacol. Ther. 74:288-94.

553 Gibson T, Weinstein S, Pfeiffer R, et al. (2011) Pre- and postfortification intake of 554 folate and risk of colorectal cancer in a large prospective cohort study in the 555 United States. Am. J. Clin. Nutr. 94: 1053-62.

556 Giovannucci E. (2004) Alcohol, one-carbon metabolism, and colorectal cancer: 557 recent insights from molecular studies. J. Nutr. 134: 2475S-81S.

558 Glynn S, Albanes D, Pietinen P, et al. (1996) Colorectal cancer and folate status: 559 a nested case-control study among male smokers. Cancer Epidemiol. 560 Biomarkers Prevent. 5: 487-94.

561 Goh Y, Bollano E, Einarson T, et al. (2007) Prenatal multivitamin 562 supplementation and rates of pediatric cancers: a meta-analysis. Clin. 563 Pharmacol. Ther. 81:685-91.

564 Gylling B, Van Guelpen B, Schneede J, et al. (2014) Low Folate Levels Are 565 Associated with Reduced Risk of Colorectal Cancer in a Population with Low 566 Folate Status. Cancer Epidemiol. Biomarkers Prev. 23: 2136-44.

567 Heinle R, Welch A. (1948) Experiments with pteroylglutamic acid and 568 pteroylglutamic acid deficiency in human leukemia [abstr]. J. Clin. Invest. 27: 569539.

570 Heresbach D, Barrioz T, Lapalus M, et al. (2008) Miss rate for colorectal 571 neoplastic polyps: a prospective multicenter study of back-to-back video 572 colonoscopies. Endoscopy 40: 284-90.

573 Hustad S, Ueland P, Vollset S, et al. (2000) Riboflavin as a determinant of 574 plasma total homocysteine: effect modification by the MTHFR C677T 575 polymorphism. Clin. Chem. 46: 1065-71. 
576 Jaszewski, R., Misra, S., Tobi, M., et al. (2008) Folic acid supplementation 577 inhibits recurrence of colorectal adenomas: a randomized chemoprevention trial.

578 World J. Gastroenterol. 14: 4492-8.

579 Johansson M, Relton C, Ueland, P, et al. (2010) Serum B vitamin levels and risk 580 of lung cancer. JAMA. 303: 2377-85.

581 Joubert B, den Dekker H, Felix J, et al. (2016) Maternal plasma folate impacts 582 differential DNA methylation in an epigenome-wide meta-analysis of newborns. 583 Nature Commun 7:10577| DOI: 10.1038/.

584 Kelly P, McPartlin J, Scott J. (1996) A combined high-performance liquid 585 chromatographic microbiological assay for serum unmetabolised folic acid. Anal. 586 Biochem. 238: 179-83.

587 Kennedy D, Stern S, Matok I, et al. (2011) Folate intake, MTHFR 588 polymorphisms, and the risk of colorectal cancer: a systematic review and meta589 analysis. J. Cancer Epidemiol. 35: 2-10.

590 Kim, Y-I., Salomon R, Graeme-Cook F, et al. (1996) Dietary folate protects 591 against the development of macroscopic colonic neoplasia in a dose responsive 592 manner in rats. Gut 39: 732-40.

593 Kim, Y-I. (2003) Role of folate in colon cancer development and progression. J. 594 Nutr. 133 (11 Suppl 1): 3731S-39S.

595 Kim Y-I. (2004) Folate, colorectal carinogenesis, and DNA methylation: lessons 596 from animal studies. Environ. Mol. Mutagen 44: 10-25.

597 Knock E, Deng L, Wu Q, et al. (2008) Strain differences in mice highlight the role 598 of DNA damage in neoplasia induced by low dietary folate. J. Nutr. 138: 653599658.

600 Kotsopoulos, J., Medline, A., Renlund, R., et al. (2005) Effects of dietary folate 601 on the development and progression of mammary tumors in rats. 602 Carcinogenesis. 26:1603-12.

603 Lajous, M., Lazcano-Ponce, E., Hernandez-Avila, M., Willett, W., Romieu, I. 604 (2006) Folate, vitamin B6, and vitamin B12 intake and the risk of breast cancer 605 among Mexican women. Cancer Epidemiol. Biomarkers Prev. 15: 443-8.

606 Lambrot R, Xu C, Saint-Phar S, et al. (2013) Low paternal dietary folate alters the 607 mouse sperm epigenome and is associated with negative pregnancy outcomes.

608 Nat. Commun. 4:2889.

609 Larsson S, Giovannucci E, Wolk A. (2006) Folate intake, MTHFR 610 polymorphisms, and risk of esophageal, gastric, and pancreatic cancer: a meta611 analysis. Gastroenterology. 131: 1271-83. 
612 Larsson S, Giovannucci E, Wolk A. (2007) Folate and risk of breast cancer: a 613 meta-analysis. J. Natl. Cancer Inst. 99: 64-76.

614 Lee J, Wei E, Fuchs C, et al. (2012) Plasma folate, methylenetetrahydrofolate 615 reductase (MTHFR), and colorectal cancer risk in three large nested case616 control studies. Cancer Causes Control 23: 537-45.

617 LeLeu R, Young G, McIntosh G. (2000) Folate deficiency reduces the 618 development of colorectal cancer in rats. Carcinogenesis 21: 2261-5.

619 Lewis S, Harbord R, Harris R, et al. (2006) Meta-analyses of observational and 620 genetic association studies of folate intakes or levels and breast cancer risk. J. 621 Natl. Cancer Inst.98: 1607-22.

$622 \mathrm{Li}$ E. (2002) Chromatin modification and epigenetic reprogramming in 623 mammalian development. Nat. Rev. Genet. 3:662-73.

624 Lindenbaum J, Rosenberg IH, Wilson P, et al. (1994) Prevalence of cobalamin 625 deficiency in the Framingham elderly population. Am. J. Clin. Nutr. 60: 2-11.

626 Liu Z, Choi SW., Crott J, et al. (2007) Mild depletion of dietary folate combined 627 with other B-vitamins alters multiple components of the Wnt pathway in the 628 mouse colon. J. Nutr. 137: 2701-8.

629 Liu Z, Choi SW, Crott, J, et al. (2008) Multiple B-vitamin inadequacy amplifies 630 alterations induced by folate depletion in p53 expression and its downstream 631 effector MDM2. Int. J. Cancer; 123: 519-25.

632 Liu Z, Ciappio E, Crott J, et al. (2011) Combined mild inadequacies of 'one633 carbon' vitamins amplify colonic Wnt-signaling and promote intestinal 634 tumorigenesis in BAT-LacZ×Apc1638N mice. FASEB J.; 25: 3136-45.

635 Logan R, Grainge M, Shepherd V, et al. (2008) Aspirin and folic acid for the 636 prevention of recurrent colorectal adenomas. Gastroenterology. 134: 29-38.

637 Ly A, Lee H, Chen J, et al. (2011) Effect of maternal and postweaning folic acid 638 supplementation on mammary tumor risk in the offspring. Cancer Res $639 \quad 2011 ; 71: 988-97$.

640 Mason JB, Choi SW. (2005) Effects of alcohol on folate metabolism: implications 641

642

643

644

645

646 647 
648 Mason JB. (2011) Folate consumption and cancer risk: a confirmation and some 649 reassurance, but we're not out of the woods quite yet. Am. J. Clin. Nutr. 94:9656506.

651

MacFarlane A, McEntee M, Stover P. (2014) Azoxymethane-induced colon carcinogenesis in mice occurs independently of de novo thymidylate synthesis 654 capacity. J. Nutr. 144:419-24.

655

656

657

658

659

660

661

662

663

664

665

666

667

668 Paspatis GA, Karamanolis DG. (1994) Folate supplementation and adenomatous

Milne E, Royle J, Miller M, et al. (2010) Maternal folate and other vitamin supplementation during pregnancy and risk of acute lymphoblastic leukemia in the offspring. Int J Cancer 126:2690-9.

National Diet and Nutrition Survey: adults aged 19-64. Volume 4. London, United Kingdom: Her Majesty's Stationery Office, 2004. Accessed 2009. www.food.gov.uk/science/dietarysurveys/ndnsdocuments/ndns4

Orjuela M, Cabrera-Munoz L, Paul L, et al. (2012) Risk of retinoblastoma is associated with a maternal polymorphism in dihydrofolatereductase (DHFR) and prenatal folic acid intake. Cancer 118:5912-9. colonic polyps. Dis. Colon Rectum. 37: 1340-1.

Pfeiffer C, Sternberg M, Fazili Z, et al. (2015) Folate status and concentrations of serum folate form in the U.S. population. Br. J. Nutr. 113: 1965-77.

Planells E, Sanchez C, Montellano M, et al. (2003) Vitamins B6 and B12 and folate status in an adult Mediterranean population. Eur. J. Clin. Nutr. 57: 777-85.

Protiva P, Mason JB, Liu Z, et al. (2011) Altered folate availability modifies the molecular environment of the human colon: implications for colorectal carcinogenesis. Cancer Prevent. Res. 4: 530-43.

Radimer K, Bindewald B, Hughes J, et al. (2004) Dietary supplement use by US adults: data from the National Health and Nutrition Examination Survey, 19992000. Am. J. Epidemiol. 160: 339-49.

688 Sabet J, Park L, lyer L, et al. (2016) Paternal B Vitamin Intake Is a Determinant 689 of Growth, Hepatic Lipid Metabolism and Intestinal Tumor Volume in Female 690 Apc1638N Mouse Offspring. PLoS One 11:e0151579. 
691 Sanjoaquin M, Allen N, Couto E, et al. (2005) Folate intake and colorectal

692

693

694

695

696

697

698

699

700

701

702

703

704

705

706

707

708

709

710

711

712

713

714

715

716

717

718

719

720

721

722

723

724

725

726

727

728

729

730

731

732

733

734

735

cancer risk: a meta-analytical approach. Int. J. Cancer 113: 825-8.

Sawaengsri H, Wang J, Reginaldo C, et al. (2016) High folic acid intake reduces natural killer cell cytotoxicity in aged mice. J. Nutr. Biochem. 30:102-7.

Schatzkin A. Intermediate markers as surrogate endpoints in cancer research. (2000) Hematol. Oncol. Clin. North Am. 14:887-905.

Schuz J, Weihkopf T, Kaatsch P. (2007) Medication use during pregnancy and the risk of childhood cancer in the offspring. Eur. J. Pediatr. 166:433-41.

Selhub J, Jacques P, Bostom A, et al. (1996) Relationship between plasma homocysteine, vitamin status and extracranial carotid-artery stenosis in the Framingham study population. J. Nutr. 126: 1258S-65S.

Shpitz B, Bomstein Y, Mekori Y, et al. (1997) Proliferating cell nuclear antigen as a marker of cell kinetics in aberrant crypt foci, hyperplastic polyps, adenomas, and adenocarcinomas of the human colon. Am. J. Surg. 174: 425-30.

Sie K, Chen J, Sohn K, et al. (2009) Folic acid supplementation provided in utero and during lactation reduces the number of terminal end buds of the developing mammary glands in the offspring. Cancer Lett 280: 72-7.

Sie K, Medline A, van Weel J, et al. (2011) Effect of maternal and postweaning folic acid supplementation on colorectal cancer risk in the offspring. Gut 60:168794.

Soetikno R, Kaltenbach T, Rouse R, et al. (2008) Prevalence of nonpolypoid (flat or depressed) colorectal neoplasms in asymptomatic and symptomatic adults. JAMA. 299: 1027-35.

Sohn K, Stempak J, Reid S,et al. (2003) The effect of dietary folate on genomic and p53-specific DNA methylation in rat colon. Carcinogenesis 24: 81-90.

Song J, Medline A, Mason JB, et al. (2000a) Effects of dietary folate on intestinal tumorigenesis in the apcMin mouse. Cancer Res. 60: 5434-40.

Song J, Sohn K, Medline A, et al. (2000b) Chemopreventive effects of dietary folate on intestinal polyps in Apc+/Msh2 mice. Cancer Res. 60: 3191-9.

Stevens V, McCullough M, Sun J, et al. High levels of folate, from supplements and fortification, are not associated with increased risk of colorectal cancer. (2011) Gastroenterology 141:98-105. 
Stolzenberg-Solomon R, Chang S, Leitzmann M, et al. (2006) Folate intake, alcohol use, and postmenopausal breast cancer risk in the Prostate, Lung,

762

763

764

Sweeney M, McPartlin J, Weir D, et al. (2003) Measurements of subnanomolar concentrations of unmetabolised folic acid in serum. J. Chromatogr. 788: 187-91.

Sweeney M, McPartlin J, Weir D, et al. (2006) Post-prandial serum folic acid response to multiple doses of folic acid in fortified bread. Br. J. Nutr. 94: 1-8.

Sweeney, M, McPartlin J, Scott J. (2007) Folic acid fortification and public health: report on threshold doses above which unmetabolised folic acid appear in serum. BMC Public Health 7: 41.

Thompson JR, Gerald PF, Willoughby ML, et al. (2001) Maternal folate supplementation in pregnancy and protection against acute lymphoblastic leukaemia in childhood: a case-control study. Lancet 358:1935-40.

Troen A, Mitchell B, Sorensen B, et al. (2006) Unmetabolized folic acid in plasma is associated with reduced natural killer cell cytotoxicity among postmenopausal women. J. Nutr. 136: 189-94.

Vollset SE, Clarke R, Lewington S, et al. (2013) Effects of folic acid supplementation on overall and site-specific cancer incidence during the randomised trials: meta-analyses of data on 50,000 individuals. Lancet 381(9871):1029-36.

Wargovich M, Chen C, Jimenez A, et al. (1996) Aberrant crypts as a biomarker for colon cancer: evaluation of potential chemopreventive agents in the rat. Cancer Epidemiol. Biomarkers Prev. 5: 355-60.

Waterland R, Jirtle R. (2003) Transposable elements: targets for early nutritional effects on epigenetic gene regulation. Mol. Cell. Biol. 23:5293-300.

Wien TN, Pike E, Wisløff T, et al. (2012) Cancer risk with folic acid supplements: a systematic review and meta-analysis. BMJ Open 2:e000653.doi:10.1136/

Wu K, Helzlsouer K, Comstock G, et al. (1999) A prospective study on folate, B12, and PLP and breast cancer. Cancer Epidemiol. Biomarkers Prev. 8: 209-17.

Wu K, Platz, E, Willett W, et al. (2009) A randomized trial on folic acid supplementation and risk of recurrent colorectal adenoma. Am. J. Clin. Nutr. 90: 1623-31.

Zhang S, Willett W, Selhub J, et al. (2003) Plasma folate, vitamin B6, vitamin B12, homocysteine, and risk of breast cancer. J. Natl. Cancer Inst. 95: 373-80. 